\title{
Anomalous roughening in experiments of interfaces in Hele-Shaw flows with strong quenched disorder
}

\author{
Jordi Soriano, * Jordi Ortín, and A. Hernández-Machado \\ Departament d'Estructura i Constituents de la Matèria, Universitat de Barcelona, Avenida Diagonal, 647, E-08028 Barcelona, Spain
}

(Received 2 August 2002; revised manuscript received 21 February 2003; published 21 May 2003)

\begin{abstract}
We report experimental evidence of anomalous kinetic roughening in the stable displacement of an oil-air interface in a Hele-Shaw cell with strong quenched disorder. The disorder consists of a random modulation of the gap spacing that is transverse to the growth direction (tracks). Experiments were performed by varying the average interface velocity and the gap spacing, and measuring the scaling exponents. The following values of the scaling exponents were obtained; $\beta \simeq 0.50, \beta^{*} \simeq 0.25, \alpha \simeq 1.0, \alpha_{l o c} \simeq 0.5$, and $z \simeq 2$. When there is no fluid injection, the interface is driven solely by capillary forces, and a higher value of $\beta$ of approximately $\beta$ $=0.65$ is measured. The presence of multiscaling and the particular morphology of the interfaces, characterized by large height differences that follow a Lévy distribution, confirms the existence of anomalous scaling. From a detailed study of the motion of the oil-air interface, we show that the anomaly is a consequence of different local velocities on the tracks plus the coupling in the motion between neighboring tracks. The anomaly disappears at high interface velocities, weak capillary forces, or when the disorder is not sufficiently persistent in the growth direction. We have also observed the absence of scaling when the disorder is very strong or when a regular modulation of the gap spacing is introduced.
\end{abstract}

DOI: 10.1103/PhysRevE.67.056308

PACS number(s): 47.55.Mh, 68.35.Ct, 05.40.-a

\section{INTRODUCTION}

The study of the morphology and dynamics of rough surfaces and interfaces in disordered media has been a subject of much interest in recent years [1], and is an active field of research with relevance in technological applications and materials characterization. These surfaces or interfaces are usually self-affine, i.e., statistically invariant under an anisotropic scale transformation. In many cases, the interfacial fluctuations follow the dynamic scaling of Family-Vicsek (FV) [2], which allows a description of the scaling properties of the interfacial fluctuations in terms of a growth exponent $\beta$, a roughness exponent $\alpha$, and a dynamic exponent $z$. However, many growth models [3-8], experiments of fracture in brittle materials, such as rock [9] or wood $[12,10,11]$, as well as experiments on sputtering [13], molecular beam epitaxy [14], and electrodeposition [15] have shown that the FV scaling is limited, and a new ansatz, known as anomalous scaling $[7,16,17]$, must be introduced. The particularity of the anomalous scaling is that local and global interfacial fluctuations scale in a different way, thus, in general, the global growth and roughness exponents $\beta$ and $\alpha$, respectively, differ from local exponents $\beta^{*}$ and $\alpha_{l o c}$.

An interesting observation from the experiments on fracture in wood, where tangential and radial fractures were studied [12], is that the morphology of the interfaces for the former were characterized by large slopes, in contrast to the smoother appearance of the latter. As pointed out by López et al. [9], the origin of anomalous scaling in these experiments seems to be related to these large slopes in the morphology of the interfaces.

In a previous work [18], we presented an experimental

*Electronic address: soriano@ecm.ub.es study of forced fluid invasion in a Hele-Shaw cell with quenched disorder. We focused on the limit of weak capillary forces and studied different types of disorder configurations at different interface velocities and gap spacings. We obtained $\beta \simeq 0.50$, which was almost independent of the experimental parameters. However, the measured roughness exponents were dependent on the experimental parameters, and two regimes were characterized, $\alpha_{1}$ at short length scales and $\alpha_{2}$ at long length scales. We also observed that in conditions of totally persistent disorder in the direction of growth, and for sufficiently strong capillary forces, a new regime emerged. This new regime must be described within the framework of anomalous scaling and was discussed in a recent paper [19]. The scaling exponents were obtained for a specific set of experimental parameters, and the range of validity of the anomaly was explored. We also introduced a phenomenological model that reproduces the interface dynamics observed experimentally and the scaling exponents.

The objective of the present paper is to analyze the experimental evidence of anomalous scaling in a systematic way, introducing additional techniques for the detection of anomalous scaling, such as multiscaling and the statistical distribution of height differences. The experimental range of anomalous scaling is studied in depth, and we investigate the effects of average interface velocity, gap spacing, and disorder configuration. We also consider the situation in which the interface is solely driven by capillary forces, which allows the behavior of the interface to be better understood at the disorder scale and its relationship with the scaling exponents at particular limits of the experiments.

The outline of the paper is as follows. In Sec. II we review the anomalous scaling ansatzs. In Sec. III, we introduce the experimental setup and describe the methodology used in data analysis. The main experimental results are presented and analyzed in Sec. IV. The discussion and final conclusions are given in Sec. V. 


\section{SCALING ANSATZS}

The statistical properties of a one-dimensional interface defined by a function $h(x, t)$ (interface height at position $x$ and time $t$ ) are usually described in terms of the fluctuations of $h$. If we consider an interface of lateral size $L$, the rms width calculated over a window $l<L$ is defined as $w(l, t)$ $=\left\langle[h(x, t)-\tilde{h}(t)]^{2}\right\rangle_{x}^{1 / 2}$, where $\tilde{h}(t)=\langle h(x, t)\rangle_{x}$, and \langle\rangle$_{x}$ is a spatial average in the $x$ direction. While the global width $w(L, t)$ follows the FV dynamic scaling [2], in the anomalous scaling assumption the local width $w(l, t)$ follows a different scaling given by

$$
w(l, t) \sim\left\{\begin{array}{lll}
t^{\beta} & \text { if } & t \ll l^{z} \\
l^{\alpha_{l o c}} \beta^{*} & \text { if } & l^{z} \ll t \ll L^{z} \\
l^{\alpha_{l o c}} L^{\alpha-\alpha_{l o c}} & \text { if } & L^{z} \ll t .
\end{array}\right.
$$

Here, $\beta$ and $\alpha$ are the global growth and roughness exponents, respectively. $z$ is the dynamic exponent with $\alpha=z \beta$. $\beta^{*}=\left(\alpha-\alpha_{l o c}\right) / z$ is the local anomalous growth exponent and $\alpha_{l o c}$ the local roughness exponent. The local time is given by $t_{l} \sim l^{z}$ and the saturation time by $t_{\times} \sim L^{z}$. Therefore, in the case of anomalous scaling, five scaling exponents (three of them independent) are needed to characterize the scaling behavior of the surface. It is common to define $\theta$ $=\alpha-\alpha_{l o c}$ as the anomalous exponent, which measures the degree of anomaly $[16,17]$. When $\theta=0$ and $\beta^{*}=0$ local and global scales behave in the same way, and we recover the usual FV scaling, i.e., $w(l, t) \sim t^{\beta}$ for $t \ll l^{z}$ and $w(l, t) \sim l^{\alpha}$ for $t \gg l^{z}$.

It is common to define a scaling function for the behavior of $w(l, t)$ in the form $w(l, t) / l^{\alpha}=g\left(l / t^{1 / z}\right)$. From Eq. (1), $g(u)$ is expected to scale as

$$
g(u) \sim\left\{\begin{array}{lll}
u^{-\left(\alpha-\alpha_{l o c}\right)} & \text { if } & u \ll 1 \\
u^{-\alpha} & \text { if } & u \gg 1 .
\end{array}\right.
$$

One of the implications of anomalous scaling is that the local width saturates when the system size also saturates, i.e., at times $t_{\times}$, and not at the local time $t_{l}$ as occurs in the FV scaling. It is also interesting to note that anomalous scaling can be identified in an experiment by plotting the local width $w(l, t)$ as a function of time for different window sizes. According to Eq. (1) the different curves would show a vertical shift proportional to $l^{\alpha_{l o c}}$.

The roughness exponents are usually determined by studying the power spectrum of the interfacial fluctuations, $S(k, t)=\langle H(k, t) H(-k, t)\rangle, \quad$ where $\quad H(k, t)=\Sigma_{x}[h(x, t)$ $-\tilde{h}(t)] \exp (i k x)$. While in the FV assumption, the power spectrum scales as

$$
S(k, t)=k^{-(2 \alpha+1)} s\left(k t^{1 / z}\right),
$$

where $s$ is the scaling function

$$
s(u) \sim\left\{\begin{array}{lll}
u^{2 \alpha+1} & \text { if } & u \ll 1 \\
\text { const } & \text { if } & u \gg 1,
\end{array}\right.
$$

in the anomalous scaling $s(u)$ is given by

$$
s(u) \sim\left\{\begin{array}{lll}
u^{2 \alpha+1} & \text { if } & u \ll 1 \\
u^{2 \theta} & \text { if } & u \gg 1 .
\end{array}\right.
$$

For times $t \gg t_{\times}$the power spectrum scales as $S(k, t)$ $\sim k^{-\left(2 \alpha_{l o c}+1\right)} t^{2 \theta / z}$ and not simply as $k^{-(2 \alpha+1)}$ of the ordinary scaling.

In general, one differentiates between two types of anomalous scaling: super-roughness and intrinsic anomalous scaling. Super-roughness is characterized by $\alpha_{l o c}=1$ and $\alpha$ $>1$, although the structure factor follows the ordinary scaling given by Eqs. (3) and (4). Thus, for super-roughness, the power spectrum provides information about the global roughness exponent $\alpha$. The intrinsic anomalous scaling strictly follows the scaling given by Eq. (5). This implies that the decay of the power spectrum with $k$ only gives a measure of $\alpha_{l o c}$, not of $\alpha$. Moreover, the particular temporal dependence of the scaling of the power spectrum allows identification of the existence of intrinsic anomalous scaling by the presence of a vertical shift of the spectral curves at different times. One has to be cautious with this particularity because the observation of a vertical shift, however, does not necessarily imply the existence of intrinsic anomalous scaling. The different forms of anomalous scaling are included in a generic dynamic scaling ansatz recently introduced by $\mathrm{Ra}-$ masco et al. [20].

\section{EXPERIMENTAL PROCEDURE AND DATA ANALYSIS}

\section{A. Experimental setup}

The experimental setup used here has been described previously in Refs. $[18,19]$. The setup consists of a horizontal Hele-Shaw cell that contains a pattern of copper tracks placed on a fiber glass substrate. The tracks are $d=0.06$ $\pm 0.01 \mathrm{~mm}$ high and are randomly distributed along the lateral direction $x$ without overlap, and fill $35 \%$ of the substrate. The lateral size of the unit track is $1.50 \pm 0.04 \mathrm{~mm}$, but wider tracks are obtained during design when two or more unit tracks are placed adjacently together. The separation between the fiber glass substrate and the top glass defines the gap spacing $b$, which was varied in the range $0.16 \leqslant b$ $\leqslant 0.75 \mathrm{~mm}$. A silicone oil (Rhodorsil $47 \mathrm{~V}$ ) is injected on one side of the cell at constant volumetric injection rate $Q$ and displaces the air initially present. A typical example of the morphology of an interface and its temporal evolution is shown in Fig. 1 [21].

Although the disorder made by tracks (which will be called $T 1.50$, the numerical value indicating the width of the unit track in millimeters) has been the main disorder pattern used in the experiments, other disorder configurations have been employed. These configurations are characterized by a random distribution of squares with a lateral size of 1.50 or $0.40 \mathrm{~mm}$, and will be called SQ1.50 and SQ0.40, respectively. A full description and characterization of these disorders, plus all additional details about the experimental setup and procedure can be found in Refs. $[18,19]$. 

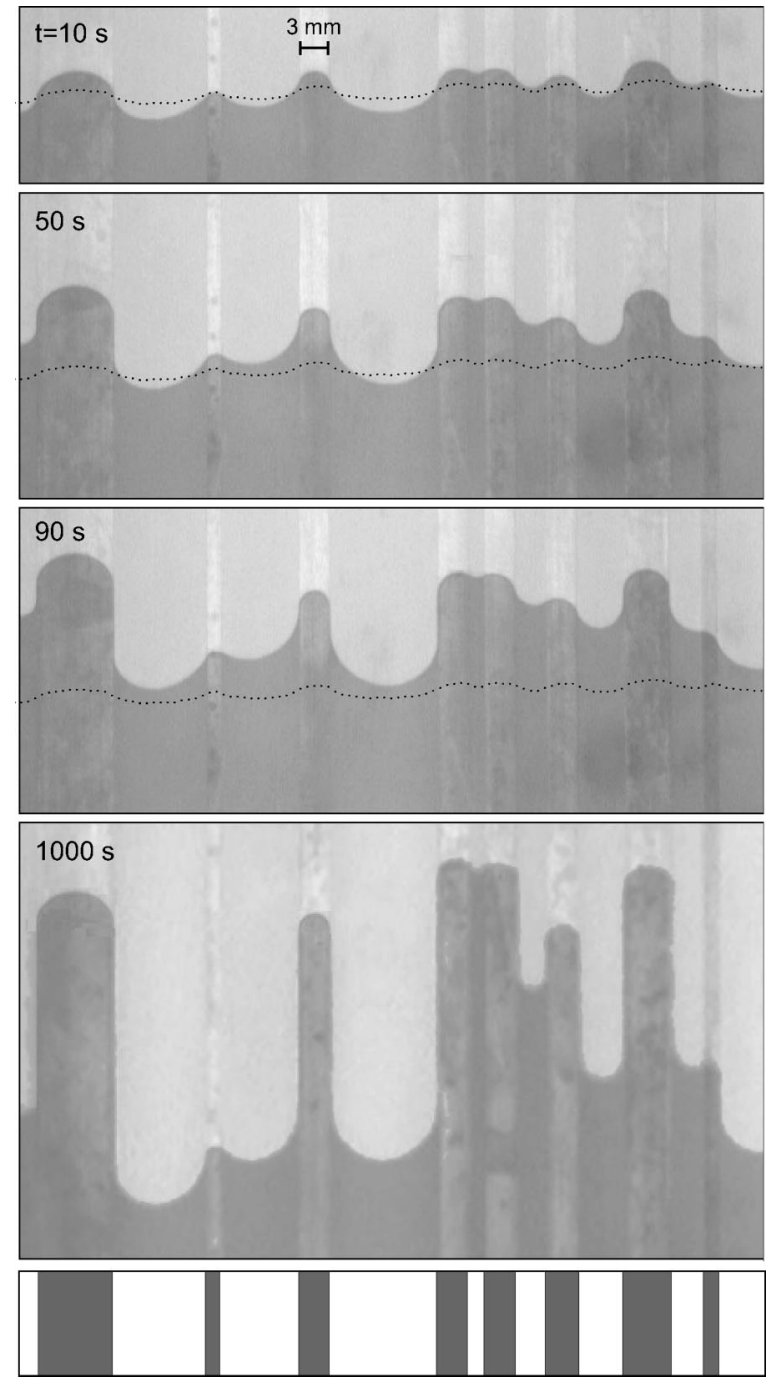

FIG. 1. Closeup views of the oil-air interface with disorder $T 1.50$. The first three pictures are early stages, while the last photo corresponds to saturation. The position of the copper tracks is shown in the lower box. The dotted line shows the position and shape of the interface profile at $t=0 \mathrm{~s}$. The experimental parameters are $v=0.08 \mathrm{~mm} / \mathrm{s}, b=0.36 \mathrm{~mm}$.

\section{B. Characterization of scaling}

As explained in Sec. II, we have studied the roughening process of the interfaces by means of the interfacial rms width $w(l, t)$ and the power spectrum $S(k, t)$. The scaling of $w(l, t)$ with time provides the global (for $l=L$ ) and local (for $l \ll L)$ growth exponents $\beta$ and $\beta^{*}$. The exponent $\beta^{*}$ has also been measured using an alternative scheme, proposed by López [22], in which the scaling of the mean local slopes with time is analyzed.

The local roughness exponents $\alpha_{l o c}$ were obtained from the scaling of the power spectrum $S(k, t)$ with $k$. The results obtained were checked using other methods, such as the dependency of the height-height correlation function $C(l, t)$ or $W(l, t)$ on the window size $l$, in both cases scaling as $l^{\alpha} l o c$. Similar results to those from $S(k, t)$ were obtained within error bars.
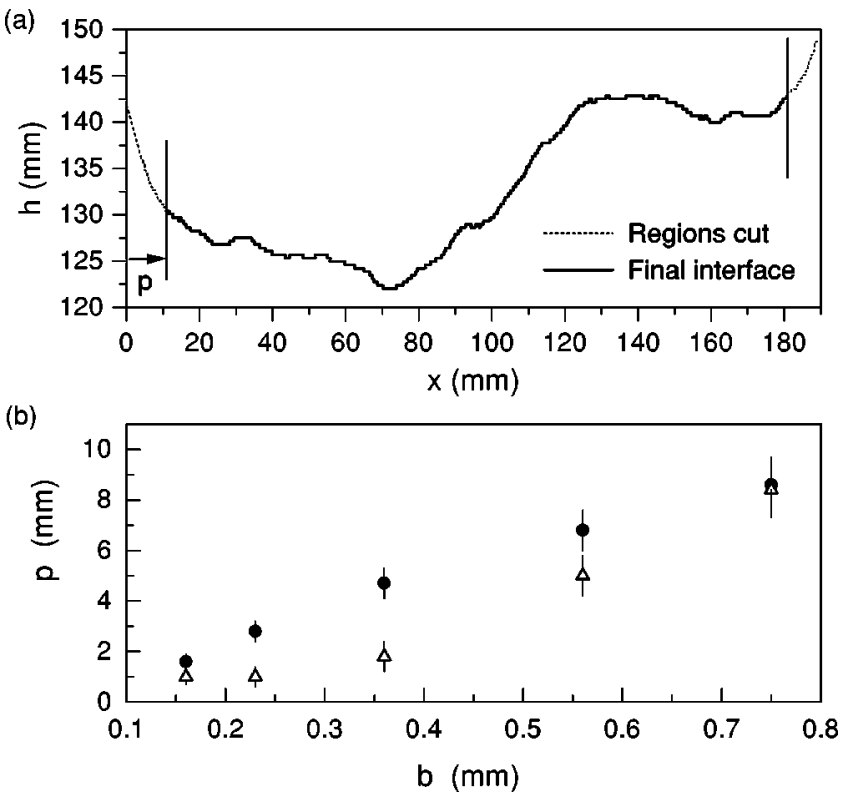

FIG. 2. (a) Example of an interface with strong side wall distortions. The dashed lines represent the regions cut at the end of the interface, and the solid line represents the interface finally studied. The experimental parameters are $v=0.08 \mathrm{~mm} / \mathrm{s}, b=0.75 \mathrm{~mm}$, and disorder SQ1.50. (b) Penetration length $p$ as a function of the gap spacing $b$ for SQ1.50 (dots) and $T 1.50$ (triangles).

Finally, within the framework of intrinsic anomalous scaling, the only direct way to measure the global roughness exponents $\alpha$, and in addition $z=\alpha / \beta$, is to study the scaling of $C(L, t)$ or $w(L, t)$ with the system size $L$. Both quantities scale as $L^{\alpha}$. Because it is not practical to perform experiments by varying the system size, we have not measured global roughness exponents directly. Instead, they have been obtained from the collapse of $S(k, t)$ or $w(l, t)$. Note that information about the value of $\alpha$ is contained in both the vertical displacement of the spectral curves with time [Eqs. (3) and (5)] and the vertical shift of the $w(l, t)$ curves for different window sizes [Eq. (1)]. The error bars that we give in the final numerical value of a particular scaling exponent take into account the various methods of analysis and the error in the different collapses.

\section{Side wall effects}

The profile $h(x, t)$, obtained from the analysis of the images, contains 515 points corresponding to a lateral size of $190 \mathrm{~mm}$. Because the oil tends to advance along the side walls of the cell, we have a distortion of the front that penetrates a distance $p$ from each side wall. This penetration length depends on the average interface velocity and is especially sensitive to variations in gap spacing. An example is presented in Fig. 2(a), which shows the effect of the distortion on an interface with experimental parameters $v$ $=0.08 \mathrm{~mm} / \mathrm{s}, b=0.75 \mathrm{~mm}$ and disorder SQ1.50.

To evaluate the effect of the distortion, we have measured the penetration length $p$ at different gap spacings $b$ for two disorder configurations SQ1.50 and T1.50. The penetration length is measured at short times, when the interface is glo- 
bally flat except at the side walls. Fitting a straight line to the central region of the interface enables us to determine $p$ as the distance between the side wall and the point where the interface deviates from the straight line. The results for the two disorder configurations analyzed are presented in Fig. 2(b), and show that $p$ increases quickly with gap spacing. For $b=0.75 \mathrm{~mm}$, the largest value studied, $p$ is about $8 \mathrm{~mm}$. Notice also that the presence of columnar disorder significantly reduces the effect of the distortion for the smallest gap spacings. To minimize the effect of the distortion, we have cut $8 \mathrm{~mm}$ from both ends of all interfaces, as shown in Fig. 2(a), reducing them to a final lateral size of $174 \mathrm{~mm}$ containing 470 pixels, which gives a data spacing of $\Delta x$ $=0.37 \mathrm{~mm}$.

\section{Data treatment}

In order to define $h(x, t)$ at the edges of the copper tracks, where (due to the finite resolution) the interface may oscillate between two neighboring pixel columns, we have taken the lowest value of the left column as first point and the highest value of the right column as second point, in the case of increasing $h$, and the highest left and lowest right in the case of decreasing $h$. This gives a finite slope to the interface at the edges of the tracks.

For data analysis convenience (particularly in the computation of the power spectrum), we have extended the number of points to $N=512$ using linear interpolation, which gives a final data spacing of $\Delta x=0.34 \mathrm{~mm}$. Although taking equally spaced points introduces an artificial cutoff on the height gradients that depends on $\Delta x$, our spatial resolution is high enough for this not to be a problem. To prove it, we have artificially increased the data spacing $\Delta x$ (by taking one pixel every $n$ pixels) and performed again the different analysis. As the number $n$ tends to 1 (and $\Delta x$ approaches the actual value used in our experiments), the results become insensitive to the spatial resolution.

Because the initial stages of roughening are so fast, the interface profile at $t=0 \mathrm{~s}$ is not a flat interface, but a profile with an initial roughness $w(0)$, as can be observed in Fig. 1 (dotted line). This roughness, on one hand, does not enable observation of the growth of the small scales at short times; on the other hand, $w(0)$ changes from one disorder configuration to another, which makes the early stages strongly dependent on the particular disorder realization. To eliminate these effects, we will always plot the subtracted width $W(l, t)$, defined as $W(l, t)=\left\langle w^{2}(l, t)-w^{2}(l, 0)\right\rangle^{1 / 2}$, where the brackets indicate average over disorder realizations. This correction is a standard procedure in the field of growing interfaces, and is reported in both experiments and numerical simulations $[1,13,23]$.

The final treatment we use is to enforce periodic boundary conditions by subtracting the straight line that connects the two ends of the interface. This procedure, well documented in the literature on kinetic roughening [24], eliminates an artificial overall slope -2 in the power spectrum due to the discontinuity at the two ends [25].

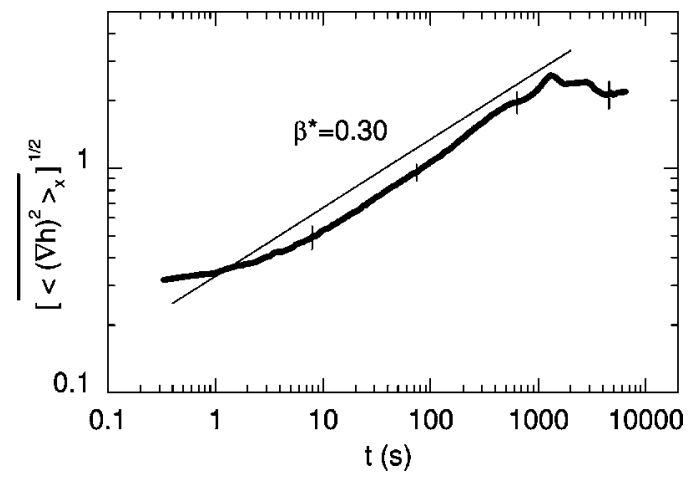

FIG. 3. Analysis of the mean local slopes as a function of time. The experimental parameters are $v=0.08 \mathrm{~mm} / \mathrm{s}, b=0.36 \mathrm{~mm}$, and disorder $T 1.50$.

\section{EXPERIMENTAL RESULTS AND ANALYSIS}

\section{A. Evidence of anomalous scaling}

The first evidence of anomalous scaling in these experiments was presented in our previous work [19] and corresponded to the experimental parameters $v=0.08 \mathrm{~mm} / \mathrm{s}$ and $b=0.36 \mathrm{~mm}$. The global and local growth exponents, measured from $W(l, t)$, were $\beta=0.52 \pm 0.02$ (corresponding to $l=L$ ) and $\beta^{*}=0.26 \pm 0.03$ (for $l=L / 256$ ). The fact that $\beta^{*}$ is clearly different from zero is robust evidence of anomalous scaling. We also studied the evolution of the power spectrum, which gave two clear power law dependences, at short times with slope $-3.4 \pm 0.1$, and at saturation with slope $-2.1 \pm 0.1$. The former was a transient regime, whose origin is the strong dominance of the capillary forces at very short times. The latter provided the local roughness exponent $\alpha_{l o c}=0.55 \pm 0.10$.

Here, we consider an alternative analysis that completes those presented in Ref. [19] and provide alternative ways to confirm the existence of anomalous scaling.

To check the reliability of the exponent $\beta^{*}$, we have studied the scaling of the mean local slopes with time, $\rho(t)$ $=\left[\overline{\left\langle(\nabla h)^{2}\right\rangle_{x}}\right]^{1 / 2}$, where the brackets denote a spatial average along $x$ and the overbar denotes average over disorder configurations. Usually $\rho(t)$ scales with time as $\rho(t) \sim t^{\kappa}$, with $\kappa<0$ for the ordinary scaling and $\kappa>0$ for anomalous scaling. In this last case, $\kappa$ is identified with the local growth exponent $\beta^{*}$. The result, presented in Fig. 3 , is $\beta^{*}=0.30$ \pm 0.05 , in good agreement with the result of $\beta^{*}$ using $W(l$ $=L / 256, t)$.

The values of $\alpha$ and $z$ were obtained in Ref. [19] by plotting $S(k, t) k^{2 \alpha+1}$ as a function of $k t^{1 / z}$. This collapse refined the numerical values of the experimental results and gave

$$
\begin{gathered}
\beta=0.50 \pm 0.04, \quad \beta^{*}=0.25 \pm 0.03, \\
\alpha=1.0 \pm 0.1, \quad \alpha_{l o c}=0.5 \pm 0.1, \quad z=2.0 \pm 0.2 .
\end{gathered}
$$

Here, the exponents $\theta$ and $\alpha$ have also been determined by means of the scaling function defined in Eq. (2). This collapse, shown in Fig. 4, gives $\theta=\alpha-\alpha_{l o c}=0.5$ and $\alpha=1$. The presence of anomalous scaling can also be identified by 


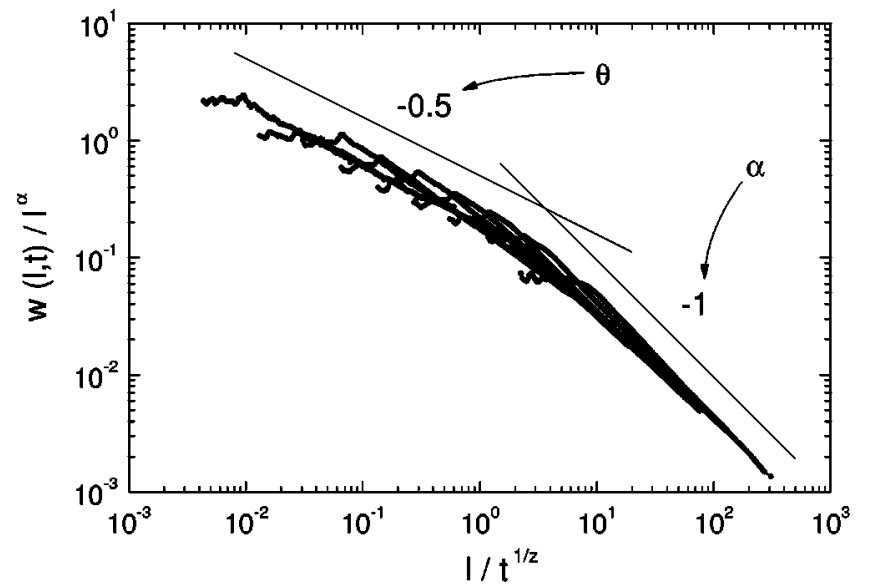

FIG. 4. Collapse of the $W(l, t)$ plots using the scaling function of Eq. (2). The experimental parameters are $v=0.08 \mathrm{~mm} / \mathrm{s}, \quad b$ $=0.36 \mathrm{~mm}$, and disorder $T 1.50$.

checking for multiscaling [4]. For this purpose, we have studied the scaling of the generalized correlations of order $q$, of the form $C_{q}(l, t)=\left\{\left\langle[h(x+l, t)-h(x, t)]^{q}\right\rangle_{x}\right\}^{1 / q} \sim l^{\alpha_{q}}$, where $\alpha_{q}$ are local roughness exponents. The results obtained are shown in Fig. 5, which gives the values of $\alpha_{q}$ at different orders $q$ for interfaces at saturation. We obtain a progressive decrease of the measured exponent, varying from $\alpha_{2} \simeq 0.58$ to $\alpha_{6} \simeq 0.25$, which confirms the existence of multiscaling in the experiment and, therefore, of anomalous scaling.

\section{B. Experimental range of anomalous scaling}

Once the existence of anomalous scaling is well characterized for a specific set of experimental parameters, we are now interested in analyzing the range of validity of the anomaly. We have performed a series of experiments by varying $v$ and fixing the gap spacing to $b=0.36 \mathrm{~mm}$ together with a series of experiments varying $b$ and fixing the average interface velocity to $v=0.08 \mathrm{~mm} / \mathrm{s}$. As we can identify the existence of anomalous scaling by looking at the behavior of

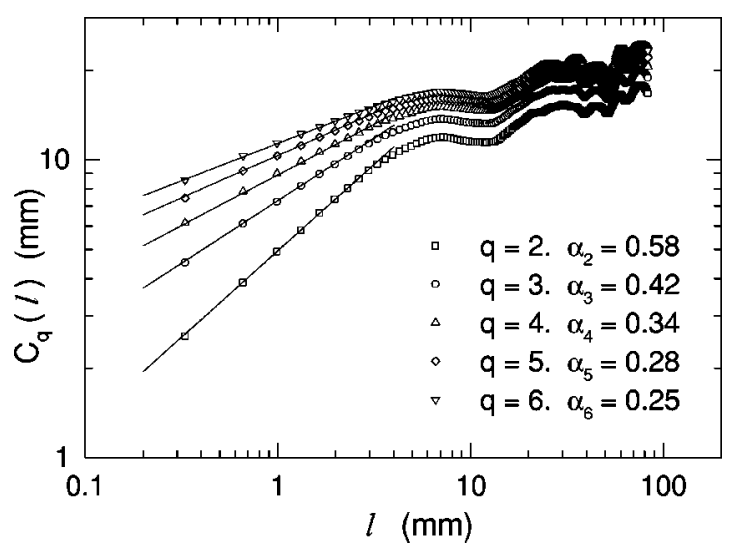

FIG. 5. Multiscaling analysis through the computation of the $q$-order height-height correlation function for interfaces at saturation. The experimental parameters are $v=0.08 \mathrm{~mm} / \mathrm{s}, \quad b$ $=0.36 \mathrm{~mm}$, and disorder $T 1.50$.

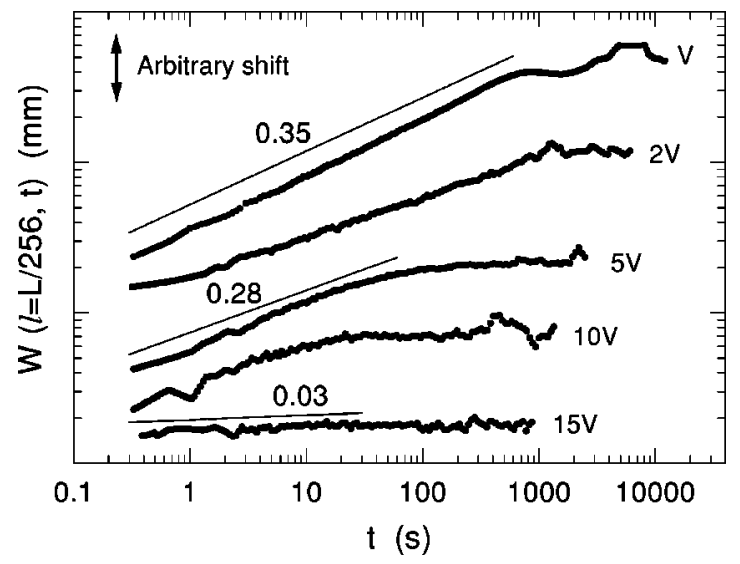

FIG. 6. $W(l, t)$ curves for a window size $l=L / 256$ and different interface velocities. The gap spacing is $b=0.36 \mathrm{~mm}$ and the disorder $T 1.50$ in all cases. $V=0.04 \mathrm{~mm} / \mathrm{s}$. The curves are vertically shifted for clarity.

the smallest scales, i.e., the existence of a $\beta^{*} \neq 0$, we present the results by plotting the local growth exponent $\beta^{*}$ for the different experiments.

\section{Interface velocity}

The results of $\beta^{*}$ at different $v$ are presented in Fig. 6 . For a wide range of interface velocities, from $V$ to $10 \mathrm{~V}$, where $V=0.04 \mathrm{~mm} / \mathrm{s}$ is the minimum velocity investigated and will be used as a reference velocity, the measured exponent $\beta^{*}$ is not very sensitive to changes in the velocity and it varies from $0.35 \pm 0.04$ for velocity $V$ to $0.28 \pm 0.04$ for velocity $10 \mathrm{~V}$. For velocities $v \gtrsim 15 \mathrm{~V}$, however, the measured value of $\beta^{*}$ quickly decays to a value close to zero, indicating that above a threshold velocity (about $13 \mathrm{~V}$ for $b$ $=0.36 \mathrm{~mm}$ ) the anomaly disappears.

In terms of the power spectrum, when the velocity is varied from $V$ to $15 \mathrm{~V}$, we always observe a vertical shift of the spectral curves and the power spectrum at different times can be collapsed to obtain the set of scaling exponents. Above the threshold velocity, the power spectrum follows ordinary scaling, i.e., without vertical displacement.

\section{Gap spacing}

The results of $\beta^{*}$ at different $b$ are presented in Fig. 7. It is clear from the different plots that the exponent $\beta^{*}$ is rather sensitive to variations of the gap spacing. Three different behaviors can be observed:

(i) For small values of $b(b=0.16 \mathrm{~mm})$, where capillary forces are dominant, we obtain $\beta^{*}=0.52 \pm 0.04$, which is identical to the corresponding value of $\beta$. This similitude is discussed in Sec. IV E.

(ii) For intermediate values of $b$ (in the range $0.23 \leqslant b$ $\leqslant 0.56 \mathrm{~mm}$ ), we obtain values of $\beta^{*}$ that vary from 0.35 \pm 0.05 for $b=0.23 \mathrm{~mm}$ to $0.25 \pm 0.04$ for $b=0.56 \mathrm{~mm}$. This range of gap spacings is characterized by strong (but not dominant) capillary forces, and is the region where the anomalous scaling can be fully characterized.

(iii) For large values of $b(b \gtrsim 0.60 \mathrm{~mm})$, we obtain $\beta^{*}$ $=0.10 \pm 0.04$ for $b=0.75 \mathrm{~mm}$, and $\beta^{*}$ nearly zero for larger 


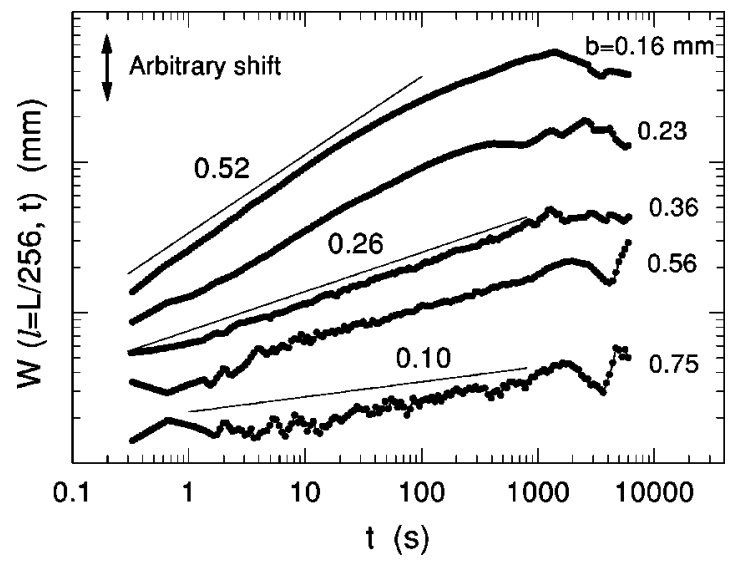

FIG. 7. $W(l, t)$ curves for a window size $l=L / 256$ and different gap spacings. The interface velocity is $v=2 V=0.08 \mathrm{~mm} / \mathrm{s}$ and the disorder $T 1.50$ in all cases. The curves are vertically shifted for clarity.

gap spacings. In this regime capillary forces are not sufficiently strong, and the anomalous scaling disappears. The analysis of $\beta^{*}$ through the scaling of the mean local slopes has given identical results within error bars, although the sensitivity of this analysis to short-time transients makes it less reliable.

As regards the power spectrum, in the range $0.23 \leqslant b$ $\leqslant 0.56$, the vertical shift of the spectral curves is present, and a regime characterized by anomalous scaling can be described. For $b=0.16 \mathrm{~mm}$, although the spectral curves are vertically shifted, a scaling regime cannot be identified. The power spectra can be collapsed, but reliable $\alpha, \alpha_{l o c}$, and $z$ exponents cannot be obtained. For $b \gtrsim 0.60 \mathrm{~mm}$, a vertical shift in the power spectra is not observed, and the scaling of the interfacial fluctuations is best described within the framework of FV ordinary scaling.

\section{Disorder properties}

Other modifications carried out to study the range of validity of the anomalous scaling are the track width and the persistence of the disorder along the growth direction. Figure 8 shows the variation of the local interfacial fluctuations for four different disorder configurations. The gap spacing and velocity have been fixed to $b=0.36 \mathrm{~mm}$ and $v=2 \mathrm{~V}$, respectively. For $T 0.40$, we obtain a similar result to that obtained with $T 1.50$, and $\beta^{*}=0.29 \pm 0.05$ was measured. However, there is evidence that the anomaly decreases as the width of the basic track is reduced, and it disappears when the width of the basic track is $\leq 0.20 \mathrm{~mm}$ due to the fact that the inplane curvature of the copper track becomes similar to the curvature of the meniscus in the $z$ direction.

Now different disorder configurations with progressively smaller persistence in the growth direction are examined. For SQ1.50, although the saturation time for the full system is $t_{\times} \simeq 1000 \mathrm{~s}$, we observe that the local interfacial fluctuations saturate at a much shorter time, which is more characteristic of ordinary scaling. However, we still observe traces of anomalous scaling at earlier times, for which $\beta^{*}=0.28$ \pm 0.04 . This is really not very surprising if we take into

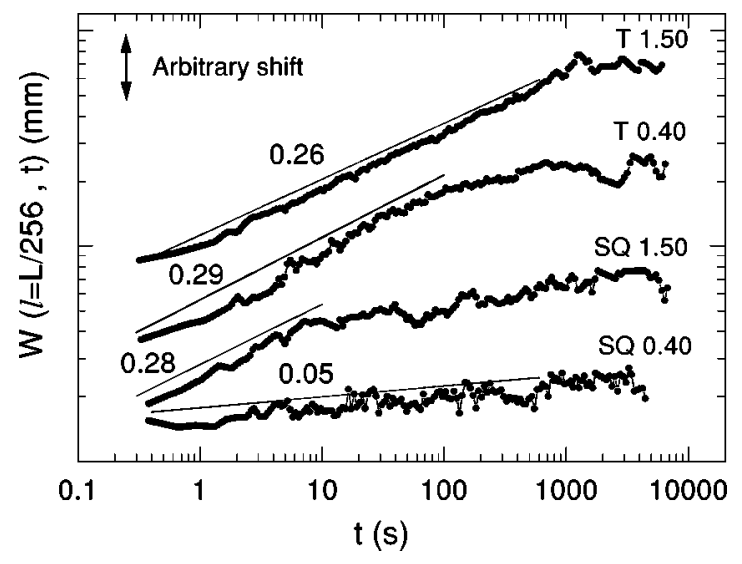

FIG. 8. $W(l, t)$ curves for a window size $l=L / 256$ and different disorder configurations with progressively shorter persistence in the growth direction. $b=0.36 \mathrm{~mm}$ and $v=2 V=0.08 \mathrm{~mm} / \mathrm{s}$ in all cases. The curves are vertically shifted for clarity.

account the fact that, for this disorder, the first array of squares is equivalent to $T 1.50$, but is only $1.50 \mathrm{~mm}$ long. Thus, at very short times, the growth follows the same behavior as tracks. With a driving velocity $0.08 \mathrm{~mm} / \mathrm{s}$, the interface takes about $10-20 \mathrm{~s}$ to cross the first array of squares, which is the same time interval for which the anomalous scaling is observed. For SQ0.40, with $t_{\times}$ $\simeq 300 \mathrm{~s}$, we measure $\beta^{*}=0.05 \pm 0.04$, indicative of the absence of anomalous scaling.

\section{Characterization by the statistical distribution of height differences}

We have also considered an alternative analysis to confirm the results of anomalous scaling. In a recent work, Asikainen et al. [26] showed that the presence of anomalous scaling can be associated with the presence of multiscaling, on one hand, and to high jumps in the morphology of the interfaces on the other. According to these authors, the distribution function of the height differences, $P(\Delta h)$, with $\Delta h \equiv\left|h\left(x_{i+1}\right)-h\left(x_{i}\right)\right|$, scales following a Lévy distribution, $P(\Delta h) \sim \Delta h^{-\gamma}$, where $0<\gamma<2$; while for ordinary scaling $P(\Delta h)$ follows a Gaussian distribution. We have checked this prediction with our experimental results. Figure 9(a) compares the shape of three different interfaces with different degrees of anomalous scaling. In all cases, $v=2 \mathrm{~V}$ and $b=0.36 \mathrm{~mm}$. All the interfaces are in the saturation regime. For $T 1.50$ (the most representative case of anomalous scaling) the interface presents high jumps at the edges of the copper tracks. For T0.40 some regions present high jumps, but, in general, the interface looks smoother. This case shows anomalous scaling, but the degree of the anomaly is much weaker than the previous case. For SQ1.50, the interface is very smooth and the interfacial fluctuations are best described with the FV scaling, with only traces of anomalous scaling at very short times. The interfaces in these three cases are similar to those reported in fracture of wood by Engфy et al. in Fig. 1 of Ref. [12]. In particular, the interfaces for $T 1.50$ or $T 0.40$ look like interfaces obtained from tangential fracture in wood; both are characterized by high 

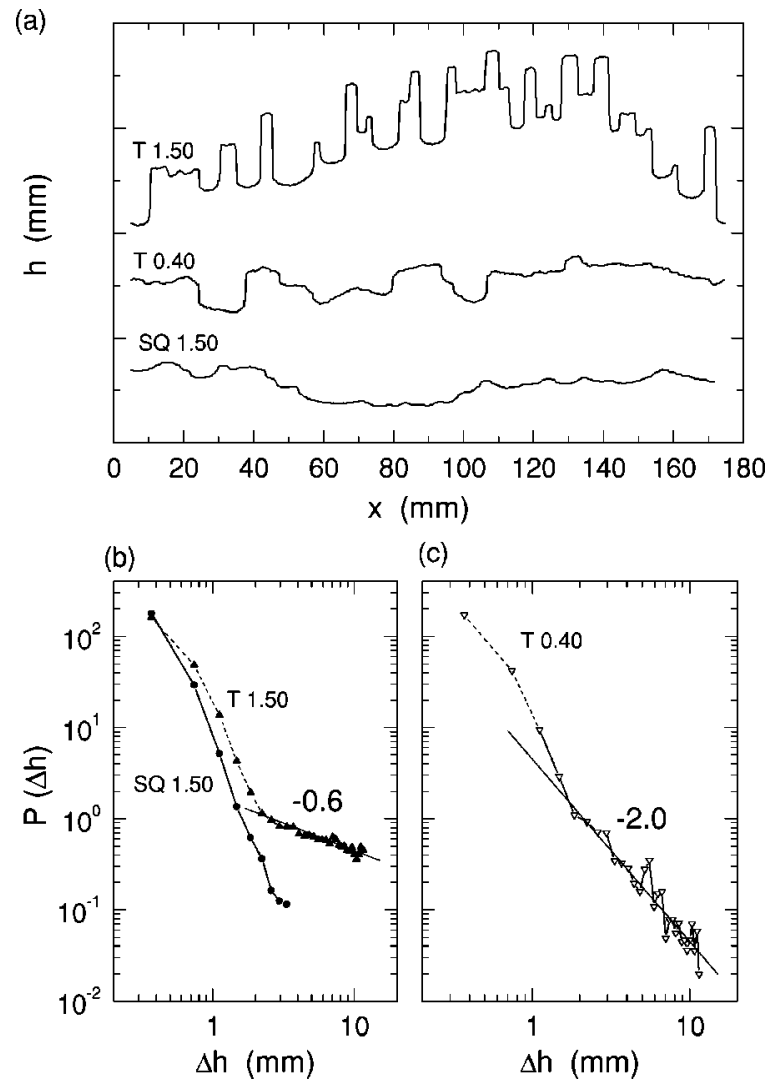

(c)

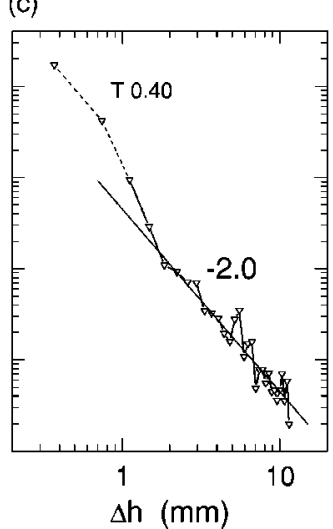

FIG. 9. (a) Samples of interfaces for different disorder configurations and identical experimental parameters $v=2 V=0.08 \mathrm{~mm} / \mathrm{s}$ and $b=0.36 \mathrm{~mm}$. (b) Distribution function $P(\Delta h)$, with $\Delta h$ $\equiv\left|h\left(x_{i+1}\right)-h\left(x_{i}\right)\right|$, for local height differences, comparing $T 1.50$ and SQ1.50. (c) The same for T0.40. See the text for details of the power law fits.

jumps and anomalous scaling. Along the same lines, the interfaces for SQ1.50 are similar to those obtained from radial fracture. These similarities indicate that the role of the wood fibres is probably equivalent to the role of the copper tracks in our system as the origin of the anomalous scaling.

The distribution functions $P(\Delta h)$ for the different interface morphologies are shown in Figs. 9(b) and 9(c). For $T 1.50$, we have two different regimes. Up to $\Delta h \simeq 2 \mathrm{~mm}$ (dashed line in the plot), we have the contribution of the height differences corresponding to the structure within tracks. For $\Delta h>2 \mathrm{~mm}$ (solid line), the contribution to the distribution function arises from the edges of the tracks. This latter contribution is responsible for the anomalous scaling, and scales with $\Delta h$ with an exponent $0.6 \pm 0.1$. A similar result is observed for T0.40. In this case, if we do not consider the contribution of the structure inside tracks (up to $1 \mathrm{~mm}$ ), we obtain a power law dependency with exponent $2.0 \pm 0.2$. The fact that the anomaly is weaker for $T 0.40$ compared with $T 1.50$ is illustrated in this case by the larger value of the slope. If we compare the distribution functions of $T 1.50$ and SQ1.50, we observe that, in the latter case, there is only a contribution from small height differences. In this case, $P(\Delta h)$ would scale with an exponent 3.6, far from the values expected for anomalous scaling.

\section{Experiments at $Q=0$}

The previous results indicate that a more detailed study on the role of capillary forces at the disorder scale has to be carried out in order to understand the origin of the anomalous scaling. For this reason, we have performed experiments at $Q=0$, where interfacial growth is driven solely by capillary forces.

The experiments at $Q=0$ were performed by initially driving the interface at high velocity up to the center of the cell. The injection is then stopped and the experiment started. We studied five gap spacings, in the range $0.16 \leqslant b$ $\leqslant 0.75 \mathrm{~mm}$, and characterized the interface velocity on copper tracks and fiber glass channels. For small gap spacings, up to $b \simeq 0.36 \mathrm{~mm}$, the morphology of the interface adopts a fingerlike configuration, which ultimately causes the interface to pinch-off at long-time intervals. For this reason, we have reduced the duration of the experiments to $1500 \mathrm{~s}$ for all gap spacings.

Figure 10 shows a comparison of the interfaces at $t$ $=500 \mathrm{~s}$ with different gap spacings. The horizontal line represents the average position of the interface at $t=0$. For $b$ $=0.16 \mathrm{~mm}$, the points of the interface on the different tracks move almost independently, with weak coupling between neighboring tracks: the oil on copper tracks always advances, while the oil on fiber glass channels, except for some particular locations, always recedes, driven by the different capillary forces associated with the different curvature of the menisci on copper tracks and the fiber glass substrate. As we increase the gap spacing, the coupling in the motion of the interface tips on copper tracks with neighboring tracks is progressively more intense. For $b=0.36 \mathrm{~mm}$, the advance on a specific copper track is coupled with its neighbors. The oil advances or recedes on a given track or channel depending on the particular disorder realization. Only the widest copper tracks or fiber glass channels have an independent motion. For $b=0.56 \mathrm{~mm}$, the coupling reaches larger regions of the cell, and for $b=0.75$ the capillary forces are no longer sufficient to allow a large deformation of the interface.

It is also noticeable that the average position of the interface does not remain at rest. Indeed, it advances in the same direction as the oil on the copper tracks. This is a consequence of the three-dimensional nature of the experiment. The total mass of fluid in the cell is conserved, but not the area measured on the images. However, the measured velocity of the advance of the interface is very low. For $b$ $=0.36 \mathrm{~mm}$, for example, the velocity is about $V / 10$.

The first analysis that we have performed with the experiments at $Q=0$ is to study the growth of interfacial fluctuations with time. Figure 11(a) shows the $W(L, t)$ curves for four different gap spacings. We observe power laws with a growth exponent $\beta$ that decreases from $0.65 \pm 0.04$ for $b$ $=0.16 \mathrm{~mm}$ to $0.50 \pm 0.04$ for $b=0.75 \mathrm{~mm}$. Clearly, when the capillary forces are sufficiently strong to allow for both the advance of the oil on the copper tracks and the recession of the oil on the fiber glass channels, we obtain a larger value of the growth exponent than in all other cases. A similar behavior is observed for $\beta^{*}$, which varies from $0.65 \pm 0.04$ 


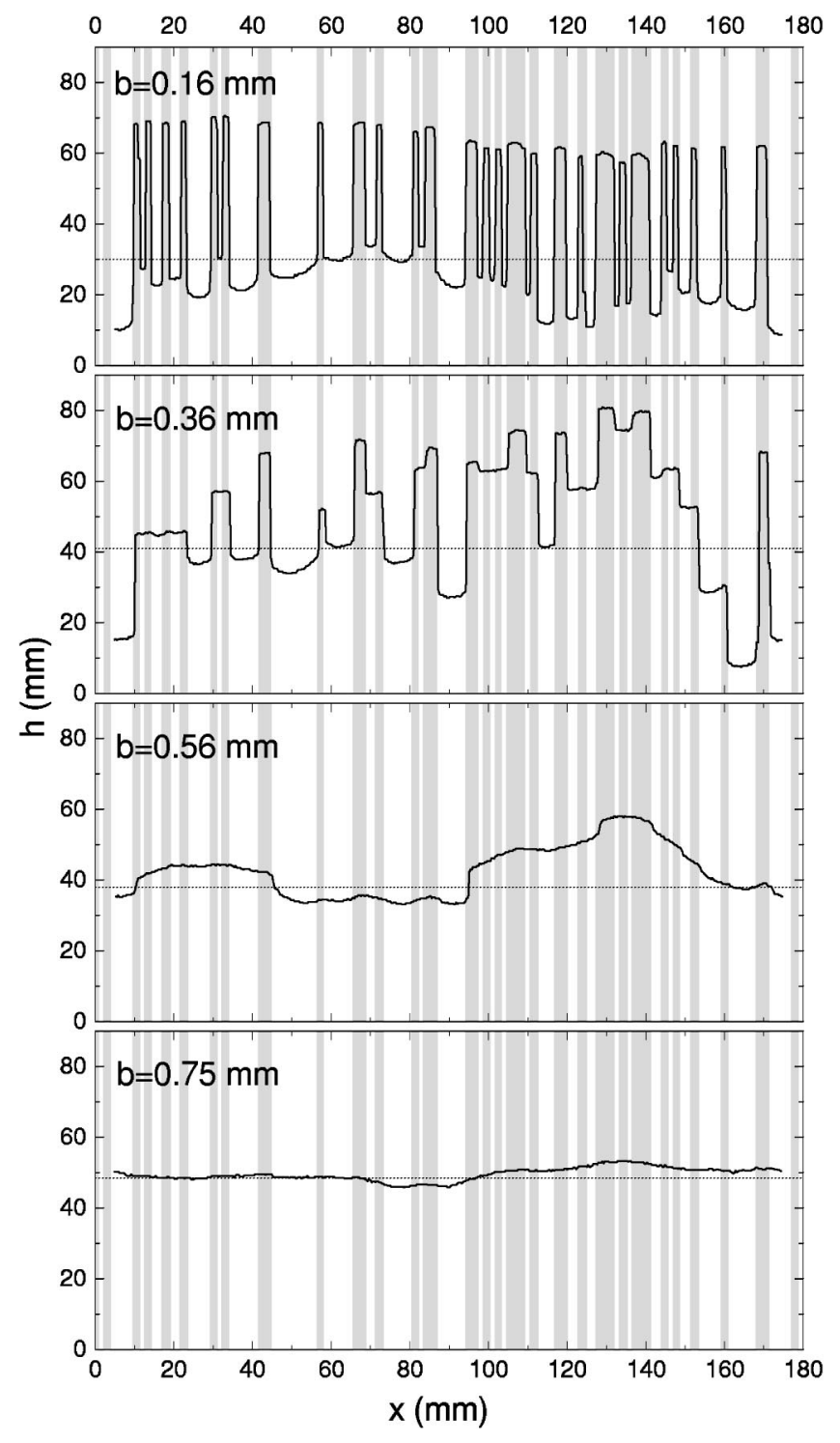

FIG. 10. Example of interfaces at $Q=0$ with different gap spacings and disorder T1.50. All the interfaces correspond to $t$ $=500 \mathrm{~s}$. The horizontal dotted line indicates the position of the interface at $t=0 \mathrm{~s}$.

for $b=0.16 \mathrm{~mm}$ to $0.15 \pm 0.05$ for $b=0.75 \mathrm{~mm}$.

As regards the behavior of the power spectrum, shown in Fig. 11(b), the fact that the experiments do not reach a saturation regime makes the analysis of the power spectrum incomplete. Qualitatively, we have observed a vertical shift of the spectral curves as time increases for gap spacings up to $b=0.56 \mathrm{~mm}$. For $b=0.75 \mathrm{~mm}$ the vertical shift is not present, confirming the absence of anomalous scaling for values of $b \geqslant 0.60 \mathrm{~mm}$ for any interface velocity. At long times, we have measured local roughness exponents that vary from $\alpha_{l o c}=0.6 \pm 0.1$ for $b=0.36 \mathrm{~mm}$ to $\alpha_{l o c}=0.9$ \pm 0.1 for $b=0.75 \mathrm{~mm}$. For $b=0.16 \mathrm{~mm}$, no clear scaling can be identified.

The second analysis that we have performed has been a detailed study of the motion of the interface on copper tracks or fiber glass channels separately. In Fig. 12 we represent $\langle h\rangle_{T}$, the mean height for those points of the interface that
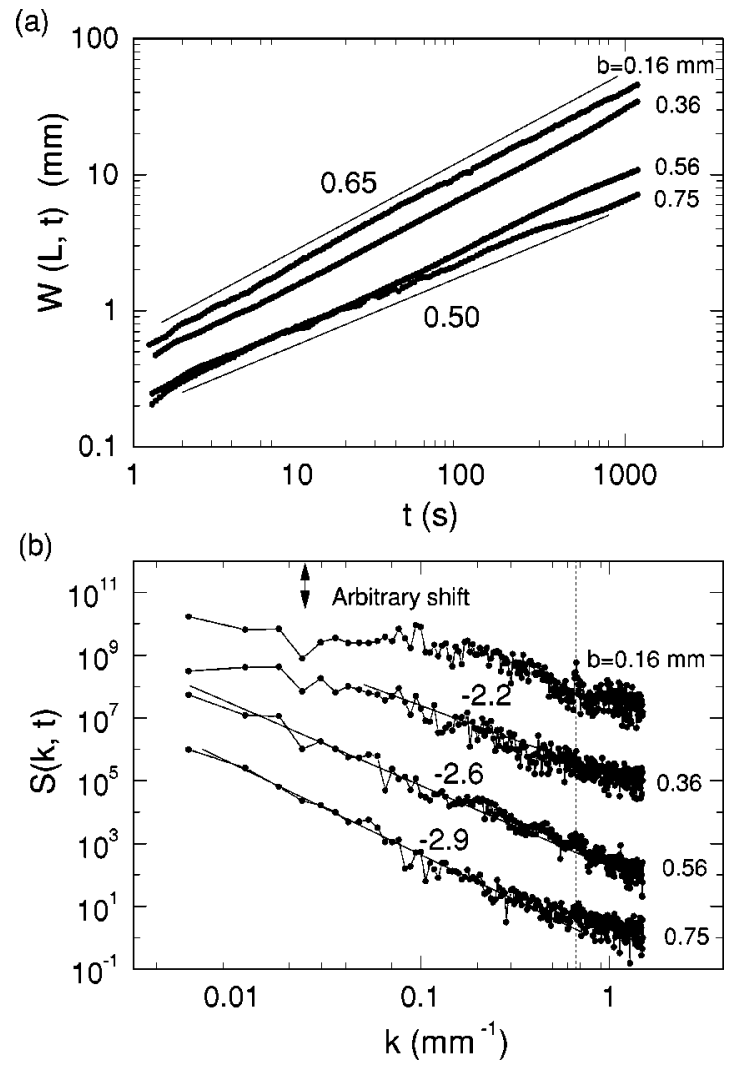

FIG. 11. Experimental results for $Q=0$ at four different gap spacings and disorder $T 1.50$. (a) Interfacial width $W(L, t)$. The straight line with slope 0.65 corresponds to a fit of the experiments with $b=0.16 \mathrm{~mm}$ and $0.36 \mathrm{~mm}$. The straight line with slope 0.50 is a fit to the experiment with $b=0.75 \mathrm{~mm}$. Each curve is an average over two disorder realizations, with two runs per disorder realization. (b) Power spectra $S(k, t)$ at $t=1000 \mathrm{~s}$. The curves have been shifted in the vertical direction for clarity. The vertical line gives the value of $k$ associated with the spatial scale of the disorder.

are advancing on copper tracks, as a function of time. For gap spacings in the range $0.16 \leqslant b \leqslant 0.36 \mathrm{~mm},\langle h\rangle_{T}$ is a power law of time with an exponent $0.50 \pm 0.03$. For larger gap spacings, the exponent is progressively smaller and the power law regime is only identifiable at short times. A similar analysis can be carried out for the fiber glass channels. In this case, we obtain the same behavior in the range 0.16 $\leqslant b \leqslant 0.36 \mathrm{~mm}$, with an exponent $0.50 \pm 0.03$. For $b$ $\geqslant 0.56 \mathrm{~mm}$, no clear scaling can be identified.

Finally, we have also studied the motion on individual tracks in order to find out the role of track width $D$. The results are shown in Fig. 13. A track is uncoupled when the motion of the interface tip on the track grows as $h \sim t^{0.5}$ independently of its neighbors' configuration. Conversely, the interface motion over a coupled track depends on the particular location of this track in the disorder realization, and the behavior $h \sim t^{0.5}$ is not observable or cannot be characterized at all times. The behavior of coupled tracks is illustrated in Figs. 14(a) and 14(b), which show two examples for $D=1.5 \mathrm{~mm}$ and $D=3.0 \mathrm{~mm}$, respectively. In both cases $b=0.36 \mathrm{~mm}$. These figures show that two tracks of the same width present different growth depending on their neighbors' 


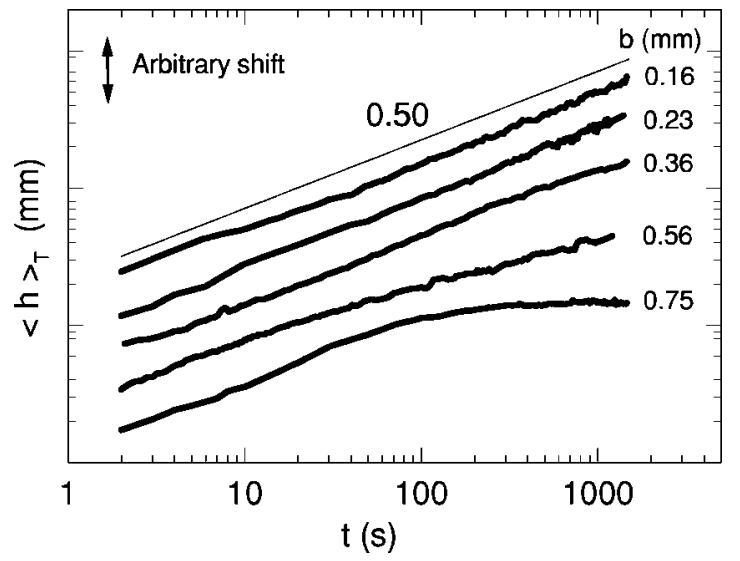

FIG. 12. Log-log plot of the average height as a function of time for the interface points advancing on copper tracks, for different gap spacings and disorder T1.50. $Q=0$ in all cases. The curves are vertically shifted for clarity.

configuration. While the uncoupled track grows as $h \sim t^{0.5}$, the coupled track follows the same behavior only at short times and afterwards reaches a stationary height. A comparison of Figs. 14(a) and 14(b) also shows that the wider the track, the weaker the coupling. The widest tracks in a given disorder realization and for $b \leqslant 0.36 \mathrm{~mm}$ remain uncoupled during all the experiment. In addition, for small gap spacings, $b \leqslant 0.16 \mathrm{~mm}$, all tracks are uncoupled.

From these studies, we conclude that it is possible to describe the interface motion on a single copper track $v_{+}^{i}$, or fiber glass channel $v_{-}^{i}$ by the simple law

$$
v_{ \pm}^{i}(t)= \pm A_{ \pm}^{i}\left(b, D_{ \pm}^{i}\right) t^{-1 / 2} \quad \text { for } \quad t \geqslant 1 \mathrm{~s},
$$

where $A_{ \pm}^{i}\left(b, D_{ \pm}^{i}\right)$ is a positive prefactor that depends on the gap spacing $b$ and the individual copper track or fiber glass channel width $D^{i}$. In general, $A^{i}$ increases with $D^{i}$ and decreases with $b$. Averaging out the prefactor $A_{ \pm}^{i}$ over different track or channel widths, we obtain a characteristic maximum velocity $v_{M \pm}$ for a given gap spacing. Equation (7) now becomes

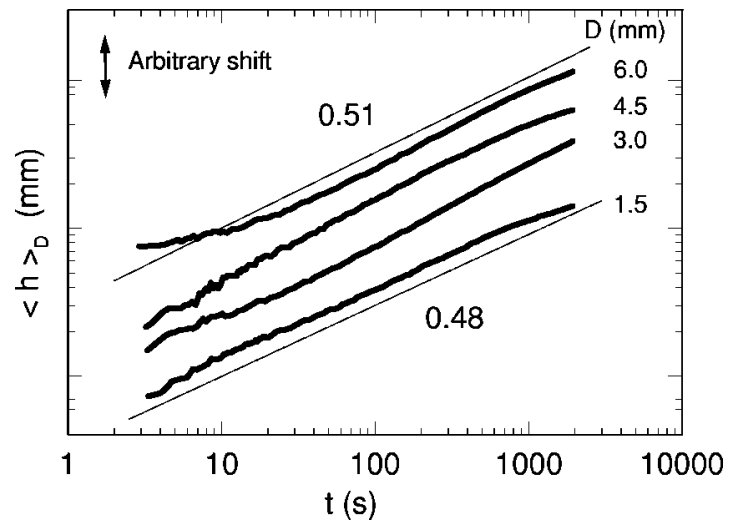

FIG. 13. Log-log plot of the average height as a function of time for the interface points advancing on uncoupled copper tracks of a specific width $D$. The experimental parameters are $Q=0, b$ $=0.36 \mathrm{~mm}$, and disorder $T 1.50$. The curves are vertically shifted for clarity. (a)

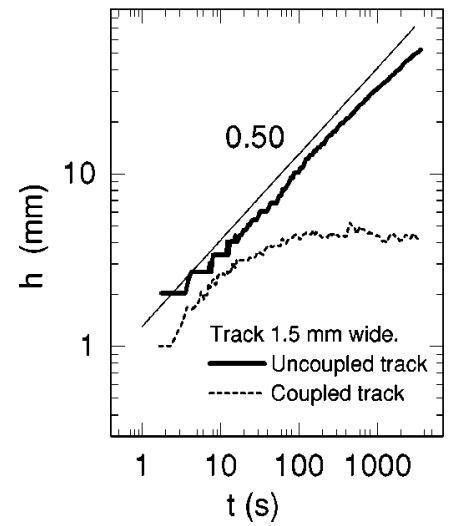

(b)

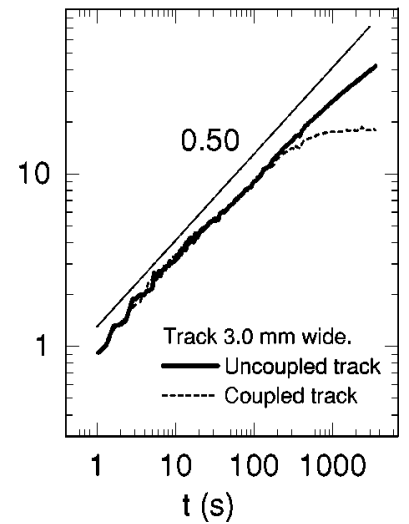

FIG. 14. Log-log plot of the average height as a function of time for the interface points advancing on a copper track of a specific width $D$. (a) $D=1.5 \mathrm{~mm}$. (b) $D=3.0 \mathrm{~mm}$. In the two cases, $Q$ $=0$ and $b=0.36 \mathrm{~mm}$.

$$
v_{ \pm}(t)= \pm v_{M \pm}(b) t^{-1 / 2} \text { for } \quad t \geqslant 1 \mathrm{~s} .
$$

Our experimental observations show that Eq. (8) is valid for gap spacings in the range $0.16 \leqslant b \leqslant 0.36 \mathrm{~mm}$. For $b$ $\geqslant 0.56 \mathrm{~mm}$, Eq. (8) only holds for copper tracks and at short times. The temporal range in which this behavior is observed goes to zero as the capillary forces become weaker.

In general, $v_{M_{+}}>v_{M_{-}}$due to the different gap spacing in copper tracks and in fiber glass channels. This difference, however, is progressively smaller as $b$ increases. For gap spacings around $b=0.36 \mathrm{~mm}$, it is reasonable to consider $v_{M_{+}} \simeq v_{M_{-}} \equiv v_{M}$. The time $t=1 \mathrm{~s}$, determined from systematic experimental observations, is a typical time in which the velocity jumps from the average interface velocity out of the disorder to $v_{M}$ inside the disorder.

\section{From $Q=0$ to low injection rates}

Equation (8) gives the velocity on copper tracks or fiber glass channels when $Q=0$ (no injection). In the presence of injection, when the interface is driven at an average interface velocity $v \neq 0$, the behavior observed experimentally is modified in the form

$$
v_{ \pm}(t)=v \pm\left(v_{M}-v\right) t^{-1 / 2},
$$

where $v_{M}=v_{M}(b)$, and $v_{+}$and $v_{-}$are the velocities averaged over copper tracks and fiber glass channels, respectively. Thus, when the interface makes contact with the disorder, the velocity on copper tracks jumps to a maximum $v_{M}$ in a characteristic time of $1 \mathrm{~s}$ and then relaxes as $t^{-1 / 2}$ to the nominal velocity. In fiber glass channels, the velocity is zero or even negative at short times, and as time goes on, the velocity increases until it reaches the nominal velocity at long times. Figure 1 illustrates this behavior. At short times, $t \simeq 10 \mathrm{~s}$, all the oil on copper tracks advances, while the oil in the widest fiber glass channel recedes. At $t \simeq 50 \mathrm{~s}$ the oil in fiber glass channels has stopped receding. At later times, the velocity at different points of the interface tends asymptotically to the nominal velocity, reached at saturation. 


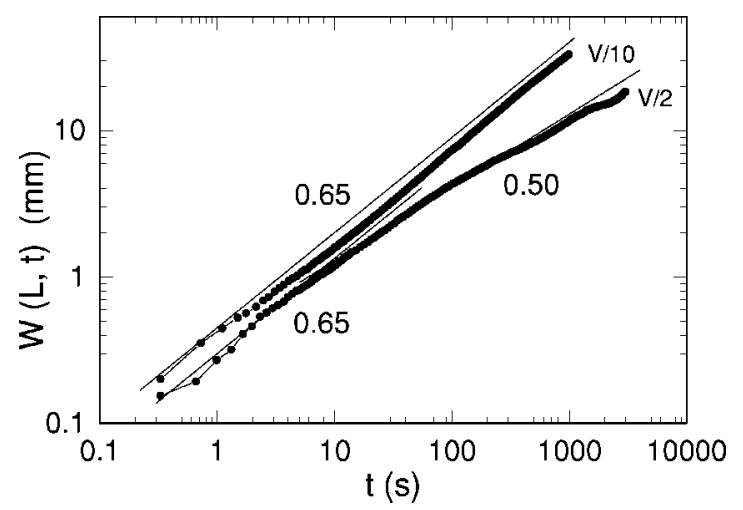

FIG. 15. Log-log plot of $W(L, t)$ for experiments at low velocity with disorder $T 1.50$. The end of the experiments is the moment at which the interfaces pinch-off. The gap spacing is $b=0.36 \mathrm{~mm}$ in both cases, and $V=0.04 \mathrm{~mm} / \mathrm{s}$. Each curve is an average over two disorder configurations, with one run per disorder configuration.

At low injection rates, the external velocity $v$ can be tuned appropriately to have an initial regime in which the oil advances on copper tracks and recedes on fiber glass channels, followed by a regime in which the oil advances at all points of the interface. This leads to two scaling regimes for $W(L, t)$, at short times with a growth exponent $\beta \simeq 0.65$, which corresponds to a regime dominated by strong capillary forces, and at longer times with a growth exponent $\beta$ $\simeq 0.50$, which corresponds to a regime where capillary and viscous forces are better balanced. We have illustrated this behavior by performing experiments at low $v$. In particular, we have studied velocities $V / 2$ and $V / 10$ using a gap spacing $b=0.36 \mathrm{~mm}$ and disorder $T 1.50$. The results are presented in Fig. 15. The difficulty of these experiments is that the interface pinches-off on the longest fingers before it reaches saturation, so we can only find out the growth regime. For $V / 2$, we observe the two power law regimes described above. For $V / 10$, only the first regime can be observed before the interface breaks. These results are qualitatively in agreement with the analysis of Hernández-Machado et al. [27], who predicted two scaling regimes, $\beta_{1}=5 / 6$ at short times (surface tension in the plane dominant) and $\beta_{2}=1 / 2$ at long times (viscous pressure dominant). However, the presence of anomalous scaling and the complicated role of capillary forces in our experiments makes a direct comparison with Ref. [27] difficult.

Another result than can be obtained from Eq. (9) is a prediction for the saturation time $t_{\times}$as a function of the average interface velocity $v$. We can estimate $t_{\times}$for a given $v$ and gap spacing $b$ by considering that saturation takes place when all tracks reach the nominal velocity $v$. This is not strictly exact due to the coupling between neighboring tracks, but it gives a good estimate of the dependency $t_{\times}(v)$, as shown in Fig. 16. For a given experiment with velocity $v<v_{M}$ the saturation takes place when the function $v_{M} t^{-1 / 2}$ crosses the nominal velocity. Thus, as shown in the inset of the figure, $t_{\times}$is expected to scale as $t_{\times} \sim v^{-2}$. This result is confirmed by our experiments with disorder $T 1.50$ and $b$ $=0.36 \mathrm{~mm}$ (open triangles in the inset).

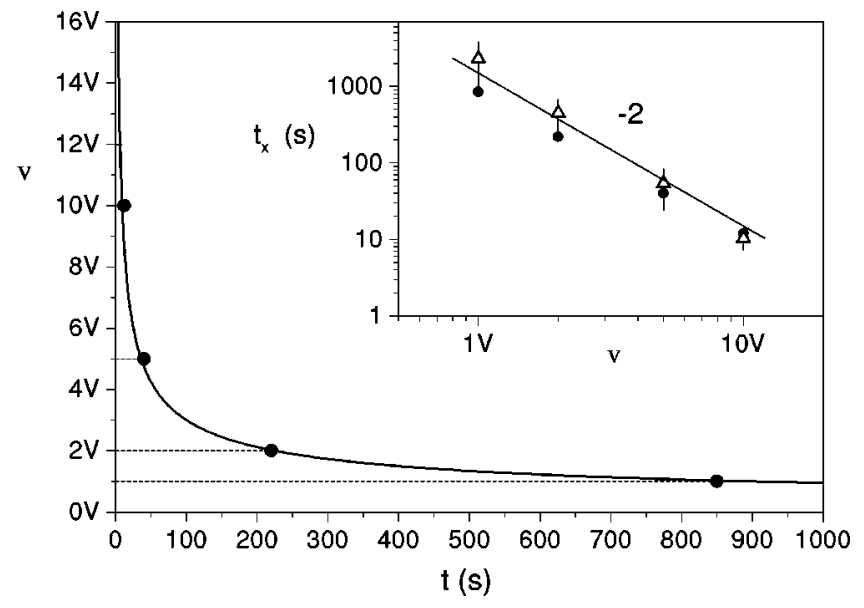

FIG. 16. Prediction of the dependency of $t_{\times}$with $v$. Main plot: for a given average velocity $v$, the system saturates when the average velocity on copper tracks decays to $v$. Inset: a log-log plot of the $t_{\times}$obtained by the previous method (filled dots) as a function of $v$ gives a straight line with slope -2 . The triangles are the experimental results for $T 1.50$ and $b=0.36 \mathrm{~mm}$.

\section{E. Experiments with a regular modulation of the gap spacing}

In order to analyze the importance of the coupling between neighboring tracks, we have performed experiments with a regular modulation of the gap spacing, consisting of copper tracks $6 \mathrm{~mm}$ wide separated by fiber glass channels 6 $\mathrm{mm}$ wide. Interface velocity and gap spacing are set to $v$ $=0.08 \mathrm{~mm} / \mathrm{s}$ and $b=0.36 \mathrm{~mm}$, respectively. The regular modulation and the temporal evolution of the resulting interfaces are presented in Fig. 17(a). Due to the symmetry of the regular pattern there is no growing correlation length along the direction of the interface. For this reason, a dynamic scaling of the interfacial fluctuations cannot even be defined. The growth of the interfacial width is due to the independent, equivalent growth of interface fingers on copper tracks, whose motion follow the $t^{-1 / 2}$ behavior [Eq. (9)]. The system saturates when the fingers reach the nominal velocity. This is shown in Fig. 17(b), which shows the evolution of $W(l, t)$ for the global $(l=L)$ and local $(l=L / 256)$ scales. The figure reveals that both scales grow in the same way. The absence of dynamic scaling is also confirmed by the power spectrum, shown in Fig. 17(c). We obtain a nearly flat spectrum, with a large peak at the value of $k$ that corresponds to the spatial periodicity of the underlying regular pattern. We also observe a vertical shift of the spectra at different times, caused by the progressive elongation of the oil fingers on copper tracks during growth.

These experiments with regular modulation of the gap spacing illustrate the need for a growing correlation length along the interface to reach a dynamic scaling regime. In a similar way to these experiments, the lateral growth of the correlation length can also be inhibited even if the modulation of the gap spacing is random, provided that the experimental parameters are such that the interface motion on the tracks is not coupled and that Eq. (9) holds for each track. Thus, for very small gap spacings $(b \leqslant 0.16 \mathrm{~mm})$ and $v$ $\geqslant V$, we observe a growth of the interfacial width with ex- 
(a)

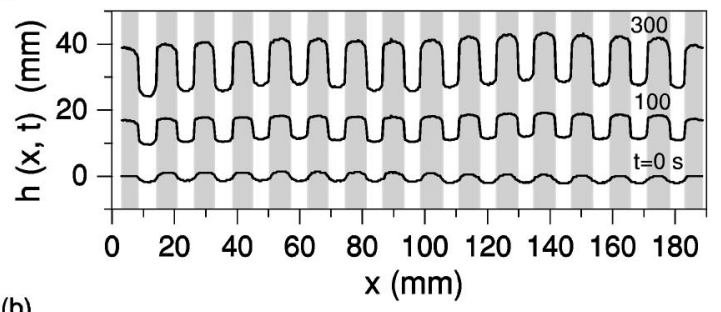

(b)

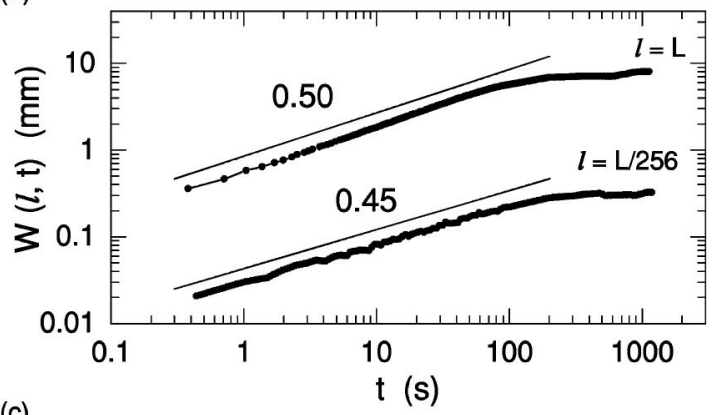

(c)

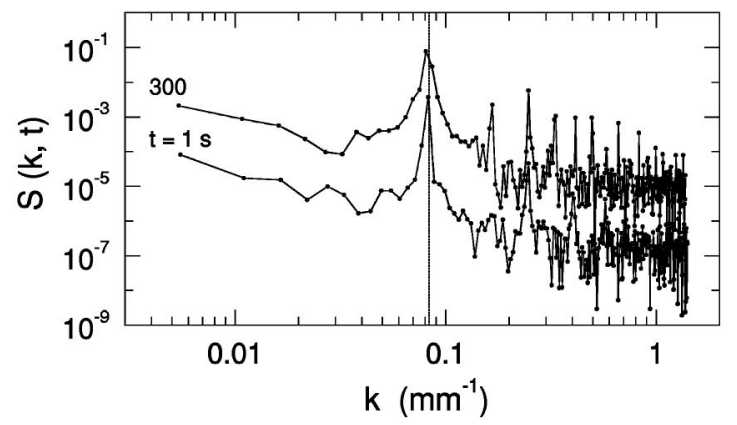

FIG. 17. Experiments with a regular modulation of the gap spacing, using tracks $6 \mathrm{~mm}$ wide. The experimental parameters are $v$ $=2 V=0.08 \mathrm{~mm} / \mathrm{s}$ and $b=0.36 \mathrm{~mm}$. (a) Temporal evolution of the interfaces. (b) $W(l, t)$ plots for global $(l=L)$ and local $(l=L / 256)$ scales. (c) Power spectrum at $t=300 \mathrm{~s}$. The vertical line indicates the value of $k$ corresponding to the spatial periodicity of the regular pattern.

ponent $\simeq 0.5$ for both global and local scales. Similarly, in the experiments at $Q=0$ with $b=0.16 \mathrm{~mm}$ the same exponent $\simeq 0.65$ is measured for both global and local scales. The power spectrum of this last case also demonstrates the lack of dynamic scaling, as can be seen in the top plot of Fig. 11(b). When the modulation of the gap spacing is regular, the transition to saturation is sharp due to the fact that all tracks have the same width, and thus all the oil fingers reach the nominal velocity at the same time. For a random pattern the transition to saturation is smooth, and does not take place strictly until the widest copper tracks (those that have the highest value of $A_{+}^{i}$ ) reach the nominal velocity. The plot for $b=0.16 \mathrm{~mm}$ of Fig. 7 illustrates this behavior.

\section{DISCUSSION AND CONCLUSIONS}

Our experimental results show that two ingredients are necessary to observe anomalous scaling in a disordered HeleShaw cell: (i) strong destabilizing capillary forces, sufficiently persistent in the direction of growth and (ii) coupling in the interface motion between neighboring tracks. These

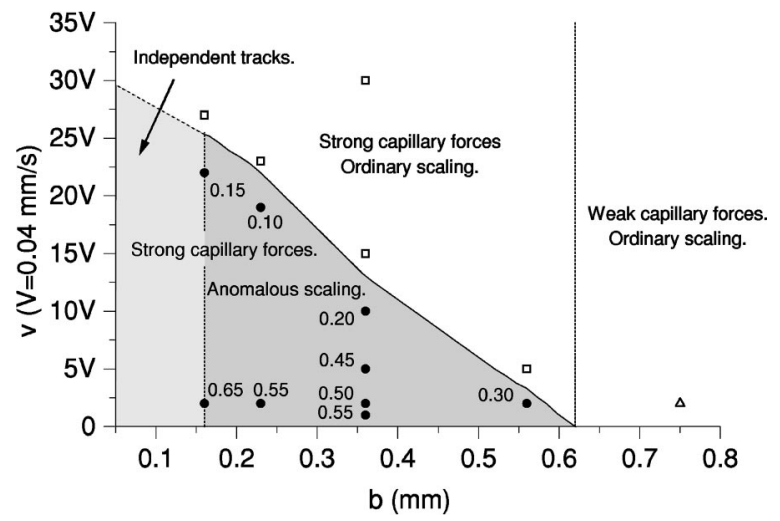

FIG. 18. Phase diagram for the anomalous scaling. The symbols indicate the parameters explored experimentally. The numbers next to the symbols in the anomalous scaling region give the value of $2 \theta / z$. The solid line represents the curve $v=v_{M}(b)$.

two ingredients give rise to large slopes at the track edges together with a growing correlation length along the interface. This scenario is represented in the phase diagram of Fig. 18, which shows the different regions of scaling explored experimentally. Each point of the diagram is an average of six experiments (three disorder configurations and two runs per disorder configuration). The dark gray area represents the region where anomalous scaling has been identified. We have indicated the value of $2 \theta / z$, which is the value needed to vertically collapse the spectral curves and, therefore, indicates the degree of anomaly. For $0.16<b$ $<0.60 \mathrm{~mm}$, and for driving velocities below $v_{M}(b)$, the capillary forces are strong enough to allow an initial acceleration and subsequent deceleration of the oil fingers on copper tracks, but are sufficiently weak to allow coupling between neighboring tracks. For $v>v_{M}$, the viscous forces become dominant, and the anomalous scaling is unobservable. For $b \geqslant 0.60 \mathrm{~mm}$, the capillary forces are not sufficiently strong and we have ordinary scaling for any velocity. Finally, for $b \leqslant 0.16 \mathrm{~mm}$ (light gray area in Fig. 18), the capillary forces are dominant, and the oil fingers on copper tracks and fiber glass channels have independent motion. In this situation, a dynamic scaling scenario is not adequate because there is no growing correlation length along the interface. $W(l, t)$ grows as a power law of time, but this is a consequence of the independent behavior for each track given by Eq. (9). Although it is not possible to determine the scaling exponents, it is still possible to perform a vertical collapse of the spectral curves because only the difference between $\alpha$ and $\alpha_{l o c}$ (i.e., $\theta$ ) is needed. This provides the values of $2 \theta / z$ for $b$ $=0.16 \mathrm{~mm}$.

In conclusion, experiments are reported of anomalous kinetic roughening in Hele-Shaw flows with quenched disorder. The displacement of an oil-air interface for different strengths of the capillary forces, flow velocity, and disorder configurations has been studied. It has been observed that scaling of the interfacial fluctuations follows the intrinsic anomalous scaling ansatz in conditions of sufficiently strong capillary forces, low velocities, and a persistent disorder in the growth direction. A random distribution of tracks has been used as representative of a persistent disorder. A differ- 
ent scaling for the global and local interfacial fluctuations has been measured, with exponents $\beta \simeq 0.50, \beta^{*} \simeq 0.25, \alpha$ $\simeq 1.0, \alpha_{l o c} \simeq 0.5$, and $z \simeq 2$. The presence of anomalous scaling has been confirmed by the existence of multiscaling. When capillary forces become dominant or when a regular track pattern is used, however, there is no growing correlation length along the interface, and therefore no dynamic scaling. A detailed study of the interface dynamics at the scale of the disorder shows that the anomaly is a consequence of the different interface velocities on copper tracks and fiber glass channels plus the coupled motion between neighboring tracks. The resulting dynamics gives interface profiles characterized by large height differences $\Delta h$ at the edges of the copper tracks. These $\Delta h$ follow an anomalous Lévy distribution characteristic of systems which display anomalous scaling.

\section{ACKNOWLEDGMENTS}

We are grateful to M. A. Rodríguez, J. Ramasco, and R. Cuerno for fruitful discussions. The research has received financial support from the Dirección General de Investigación (MCT, Spain), project BFM2000-0628-C03-01. J.O. acknowledges the Generalitat de Catalunya for additional financial support.
[1] A.-L. Barabási and H.E. Stanley, Fractal Concepts in Surface Growth (Cambridge University Press, Cambridge, 1995).

[2] F. Family and T. Vicsek, J. Phys. A 18, L75 (1985).

[3] M. Schroeder, M. Siegert, D.E. Wolf, J.D. Shore, and M. Plischke, Europhys. Lett. 24, 563 (1993).

[4] J. Krug, Phys. Rev. Lett. 72, 2907 (1994).

[5] S. Das Sarma, C.J. Lanczycki, R. Kotlyar, and S.V. Ghaisas, Phys. Rev. E 53, 359 (1996).

[6] C. Dasgupta, S. Das Sarma, and J.M. Kim, Phys. Rev. E 54, R4552 (1996).

[7] J.M. López and M.A. Rodríguez, Phys. Rev. E 54, R2189 (1996).

[8] M. Castro, R. Cuerno, A. Sánchez, and F. Domínguez-Adame, Phys. Rev. E 57, R2491 (1998).

[9] J.M. López and J. Schmittbuhl, Phys. Rev. E 57, 6405 (1998).

[10] S. Morel, J. Schmittbuhl, J.M. López, and G. Valentin, Phys. Rev. E 58, 6999 (1998).

[11] S. Morel, J. Schmittbuhl, E. Bouchaud, and G. Valentin, Phys. Rev. Lett. 85, 1678 (2000).

[12] T. Engøy, K.J. Måløy, A. Hansen, and S. Roux, Phys. Rev. Lett. 73, 834 (1994).

[13] J.H. Jeffries, J.-K. Zuo, and M.M. Craig, Phys. Rev. Lett. 76, 4931 (1996).

[14] H.-N. Yang, G.-C. Wang, and T.-M. Lu, Phys. Rev. Lett. 73, 2348 (1994).
[15] S. Huo and W. Schwarzacher, Phys. Rev. Lett. 86, 256 (2001).

[16] J.M. López, M.A. Rodríguez, and R. Cuerno, Phys. Rev. E 56, 3993 (1997).

[17] J.M. López, M.A. Rodríguez, and R. Cuerno, Physica A 246, 329 (1997).

[18] J. Soriano, J. Ortín, and A. Hernández-Machado, Phys. Rev. E 66, 031603 (2002).

[19] J. Soriano, J.J. Ramasco, M.A. Rodríguez, A. HernándezMachado, and J. Ortín, Phys. Rev. Lett. 89, 026102 (2002).

[20] J.J. Ramasco, J.M. López, and M.A. Rodríguez, Phys. Rev. Lett. 84, 2199 (2000).

[21] Real-life motion pictures of the experiments are available at http://debian01.ecm.ub.es

[22] J.M. López, Phys. Rev. Lett. 83, 4594 (1999).

[23] G. Tripathy and W. van Saarloos, Phys. Rev. Lett. 85, 3556 (2000).

[24] I. Simonsen, A. Hansen, and O.M. Nes, Phys. Rev. E 58, 2779 (1998).

[25] J. Schmittbuhl, J.-P. Vilotte, and S. Roux, Phys. Rev. E 51, 131 (1995).

[26] J. Asikainen, S. Majaniemi, M. Dubé, and T. Ala-Nissila, Phys. Rev. E 65, 052104 (2002).

[27] A. Hernández-Machado, J. Soriano, A.M. Lacasta, M.A. Rodríguez, L. Ramírez-Piscina, and J. Ortín, Europhys. Lett. 55, 194 (2001). 